Document downloaded from:

http://hdl.handle.net/10251/147746

This paper must be cited as:

Sanz Diaz, R.; García Gil, PJ.; Fridman, E.; Albertos Pérez, P. (2020). Robust Predictive Extended State Observer for a Class of Nonlinear Systems with Time-Varying Input Delay. International Journal of Control. 93(2):217-225. https://doi.org/10.1080/00207179.2018.1562204

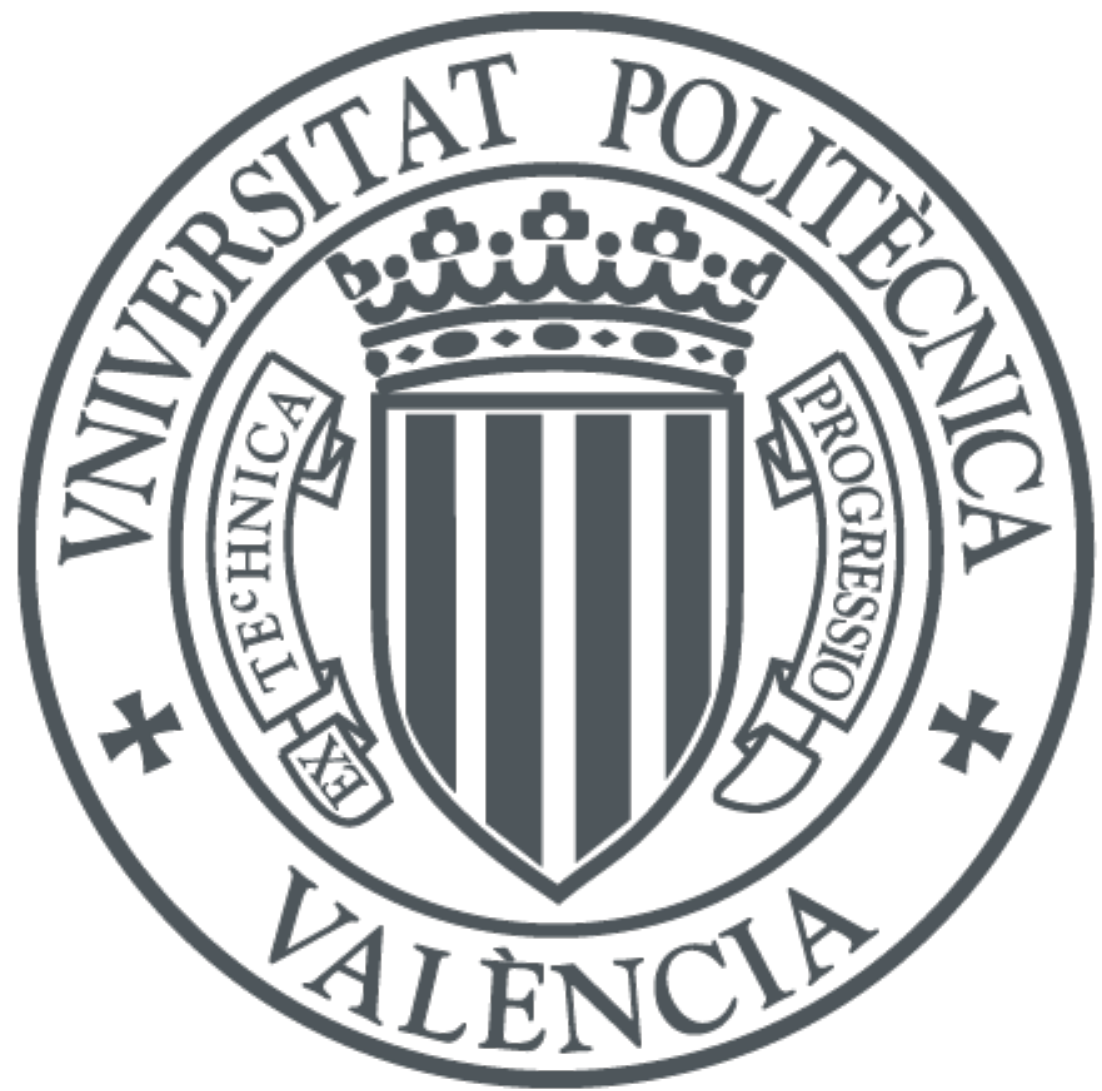

The final publication is available at

https://doi.org/10.1080/00207179.2018.1562204

Copyright Taylor \& Francis

Additional Information 


\title{
Robust Predictive Extended State Observer for a Class of Nonlinear Systems with Time-Varying Input Delay
}

\author{
R. Sanz ${ }^{\mathrm{a}}$, P. García ${ }^{\mathrm{a}}$, E. Fridman ${ }^{\mathrm{b}}$ and P. Albertos ${ }^{\mathrm{a}}$ \\ ${ }^{a}$ Department of Control Systems Engineering, Universitat Politècnica de València, 46022 \\ València, Spain; ${ }^{b}$ School of Electrical Engineering, Tel-Aviv University, Israel
}

\begin{abstract}
ARTICLE HISTORY
Compiled November 13, 2018

ABSTRACT

This paper deals with asymptotic stabilization of a class of nonlinear input-delayed systems via dynamic output-feedback in the presence of disturbances. The proposed strategy has the structure of an observer-based control law, in which the observer estimates and predicts both the plant state and the external disturbance. A nominal delay value is assumed to be known and stability conditions in terms of linear matrix inequalities are derived for fast-varying delay uncertainties. Asymptotic stability is achieved if the disturbance or the time delay are constant. The controller design problem is also addressed and a numerical example with an unstable system is provided to illustrate the usefulness of the proposed strategy.
\end{abstract}

\section{KEYWORDS}

Time-varying delay, nonlinear, output-feedback, robust control, LMI

\section{Introduction}

Time delays are ubiquitous in many engineering applications such as chemical or biological processes, oil or gas factories, and networked control. Large delays often lead to closed-loop instability if they are not taken into account and limit the achievable performance of conventional controllers (Fridman, 2014a).

When dealing with stabilization of input-delayed systems, predictive feedback plays a key role. For the linear SISO case, the stabilization problem in the presence of input/output delays can be solved by the so-called Smith Predictor (Smith, 1957), which is actually a predictive model-based controller formulated in the frequency domain. In the time domain, similar control strategies have been proposed (Artstein, 1982; Manitius \& Olbrot, 1979), even for nonlinear time-varying systems (Bekiaris-Liberis \& Krstic, 2012), all of them requiring a state predictor. However, in many cases, little attention is devoted to the predictor implementation, often assuming stability of the open-loop process. This has been a matter of concern for some researchers (Engelborghs, Dambrine, \& Roose, 2001; Mondié \& Michiels, 2003; Zhong, 2004), as the discretization of the integral terms involved may lead to instability of the closed-loop. For nonlinear systems, the implementation may be even more challenging as it requires the on-line integration of nonlinear functions. See the recent monograph (Karafyllis \& Krstic, 2017). 
In order to avoid integral terms in the control law, the idea of a predictor in observer form has been receiving increasing attention. It was first introduced in (Besançon, Georges, \& Benayache, 2007) for systems with small input delays and extended in (Najafi, Hosseinnia, Sheikholeslam, \& Karimadini, 2013; Najafi, Sheikholeslam, Wang, \& Hosseinnia, 2014) to larger delays by using the cascade observer structure initiated in (Germani, Manes, \& Pepe, 2002). The idea is to use a chain of observers so that each of them predicts the state over a fraction of the delay. This is known in the literature as the sequential predictors technique. Recently, this methodology has been exploited by some researchers (Ahmed-Ali, Cherrier, \& Lamnabhi-Lagarrigue, 2012; Léchappé, Moulay, \& Plestan, 2016; Mazenc \& Malisoff, 2016; Sanz, Garcia, Fridman, $\&$ Albertos, 2018). However, in the context of sequential predictors, nonlinearities have not been addressed in any of the aforementioned works.

Disturbance rejection is also a central issue in process control, specially challenging for time-delay systems. Several works devoted to improving disturbance rejection of state predictors have been reported recently in the literature. The inverse optimality of a filtered state predictor with respect to a functional involving the disturbance was shown in (Krstic, 2008). A filtered prediction was also considered in (Sanz, García, \& Albertos, 2017) with a frequency-domain approach. Additional delayed feedback was considered in (Léchappé, Moulay, Plestan, Glumineau, \& Chriette, 2015) to reject constant disturbances. A modified prediction based on a disturbance observer was proposed in (Sanz, Garcia, \& Albertos, 2016), leading to rejection of polynomial-in-time disturbances and also better attenuation of sufficiently smooth signals. Similar results are also reported in (Furtat, Fridman, \& Fradkov, 2018). For unknown sinusoidal disturbances, cancellation by means of adaptive control schemes have been also achieved in (Basturk, 2017; Basturk \& Krstic, 2015).

The observer structure of the sequential predictor approach makes it suitable to combine with a disturbance observer. This key idea was recently used in (Sanz, García, Fridman, \& Albertos, 2017), where nonlinearities were introduced. The previous work is extended in different directions in this paper. First, an additional nonlinear term is used in the control law in order to counteract the nonlinearity. Second, a chain of observers of arbitrary length is considered here, while only a one-element chain was considered in Sanz, García, Fridman, and Albertos (2017) for simplicity. Third, a generator model of the disturbance is considered to achieve rejection of time-varying disturbances. These modifications lead to a substantially more complicated closed-loop stability analysis. A systematic design procedure is given to compute the observer gains of all elements in the chain, as well as the feedback gain for the controller. Stability is then guaranteed in spite of the nonlinearity and the time-varying delay.

The rest of the paper is structured as follows. The problem formulation and preliminaries are given in Section 2. The proposed strategy is developed in Section 3, while the closed-loop stability and the controller design are tackled in Section 4. The main results are illustrated through a numerical example in Section 5.

\section{Preliminaries}

The present work deals with the class of input-delayed systems defined by

$$
\begin{aligned}
\dot{x}(t) & =A x(t)+B[u(t-\tau(t))+w(t)+g(t, x)], \\
y(t) & =C x(t),
\end{aligned}
$$


where $A, B, C$ are known matrices of appropriate dimensions, $x \in \mathbb{R}^{n}$ is the state, $y \in \mathbb{R}^{q}$ is the measured output and $u \in \mathbb{R}$ is the control input, $w: \mathbb{R}_{\geq 0} \rightarrow \mathbb{R}$ is an unknown external disturbance and $g: \mathbb{R}_{\geq 0} \times \mathbb{R}^{n} \rightarrow \mathbb{R}^{n}$ is a known nonlinearity. The time-varying delay is assumed to have the form

$$
\tau(t)=h+\eta(t), \quad 0 \leq \eta(t) \leq \bar{\eta},
$$

where the nominal value of the delay, $h \geq 0$, is known. The unknown time-varying function $\eta(t)$ is supposed to be piecewise-continuous and non-negative. In addition, the following assumptions are made:

Assumption 1. The pair $(A, B)$ is stabilizable and the pair $(A, C)$ is detectable.

Assumption 2. The nonlinearity has the structure $g(t, x)=g^{t}(t) g^{x}(x)$. There exist known constants $c_{1}, c_{2}>0$ such that $\left|g^{t}(t)\right| \leq c_{1}, \forall t \geq 0$ and

$$
\left|g^{t}\left(t_{1}\right)-g^{t}\left(t_{2}\right)\right| \leq c_{2}\left|t_{1}-t_{2}\right|, \forall t_{1}, t_{2} \geq 0 .
$$

Furthermore, $g^{x}(0)=0$ and there exists a known vector $m \in \mathbb{R}^{n}$ such that

$$
\left|g^{x}\left(x_{1}\right)-g^{x}\left(x_{2}\right)\right| \leq\left|l^{T}\left(x_{1}-x_{2}\right)\right|,
$$

for all $x_{1}, x_{2}$ in some region $\mathcal{D}$, containing the origin.

Assumption 3. The disturbance signal can be modeled by the exogenous system

$$
\begin{aligned}
\dot{\xi}(t) & =G \xi(t) \\
w(t) & =H \xi(t),
\end{aligned}
$$

where $G \in \mathbb{R}^{r \times r}, H \in \mathbb{R}^{1 \times r}$ are known and form a completely observable pair and $\xi \in \mathbb{R}^{r}$ is a generator vector with unknown initial condition $\xi(0)$.

The first assumption is necessary for the stabilization of (1)-(2) via dynamic output feedback. Assumption 2 basically implies Lipschitz continuity of the nonlinearity with respect to both arguments, since it is assumed to be the product of two Lipschitz functions. Assumption 3 allows to represent a variety of signals such as sinusoidal or polynomial disturbances. Let us state the following auxiliary lemma, which will be used in the stability proof.

Lemma 1. Under Assumption (2), the following holds

$$
\left|g\left(t_{1}, x_{1}\right)-g\left(t_{2}, x_{2}\right)\right| \leq c_{1}\left|l^{T}\left(x_{1}-x_{2}\right)\right|+c_{2}\left|t_{1}-t_{2}\right|\left|l^{T} x_{2}\right|,
$$

for all $t_{1}, t_{2} \geq 0$ and any $x_{1}, x_{2} \in \mathcal{D}$.

Proof. Computing the norm and adding and subtracting $g^{t}\left(t_{1}\right) g^{x}\left(x_{2}\right)$ leads to

$$
\begin{aligned}
\left|g\left(t_{1}, x_{1}\right)-g\left(t_{2}, x_{2}\right)\right| & =\left|g^{t}\left(t_{1}\right) g^{x}\left(x_{1}\right)-g^{t}\left(t_{2}\right) g^{x}\left(x_{2}\right)\right| \\
& =\left|g^{t}\left(t_{1}\right) g^{x}\left(x_{1}\right)-g^{t}\left(t_{1}\right) g^{x}\left(x_{2}\right)+g^{t}\left(t_{1}\right) g^{x}\left(x_{2}\right)-g^{t}\left(t_{2}\right) g^{x}\left(x_{2}\right)\right| \\
& \leq\left|g^{t}\left(t_{1}\right)\right|\left|g^{x}\left(x_{1}\right)-g^{x}\left(x_{2}\right)\right|+\left|g^{x}\left(x_{2}\right)\right|\left|g^{t}\left(t_{1}\right)-g^{t}\left(t_{2}\right)\right|,
\end{aligned}
$$


and thus the result follows by employing the bounds stated in Assumption 2.

The goal is to find an observer-based output-feedback control law such that the system (1)-(2) is robustly stabilized for all time-varying delays described by (3) when either the disturbance or the delay are constant. It should be remarked that asymptotic stability in the presence of both time-varying disturbances and delays is not pursued in this work, for which a delay estimation strategy would be necessary.

\section{Proposed strategy}

Let us define $z(t)=\left[x^{T}(t), \xi(t)\right]^{T} \in \mathbb{R}^{n_{z}}$ as an extended state with $n_{z}=n+r$, containing both the system state and the disturbance. Then the dynamics (1)-(2) can be rewritten as

$$
\begin{aligned}
\dot{z}(t) & =A_{z} z(t)+B_{z}[u(t-\tau(t))+g(t, x)], \\
y(t) & =C_{z} z(t),
\end{aligned}
$$

where

$$
A_{z}=\left[\begin{array}{cc}
A & B H \\
0 & G
\end{array}\right], \quad B_{z}=\left[\begin{array}{c}
B \\
0
\end{array}\right], \quad \text { and } \quad C_{z}=\left[\begin{array}{ll}
C & 0
\end{array}\right]
$$

Now, a predictive observer is adopted to obtain a future estimation of the augmented state $h$ units of time ahead. Following the ideas in (Besançon et al., 2007; Germani et al., 2002; Najafi et al., 2013), the proposed observer has a chain structure, such that each of the observer states, denoted by $\bar{z}_{j}(t)=\left[\bar{x}_{j}^{T}(t), \bar{\xi}_{j}(t)\right]^{T}$, estimates a prediction of the augmented state over a fraction of the total delay, $z\left(t+h_{j}\right)$, with $h_{j}=\frac{j}{m} h$. The proposed observer is given by

$$
\begin{aligned}
& \dot{\bar{z}}_{1}(t)=A_{z} \bar{z}_{1}(t)+B_{z}\left[u\left(t-\bar{h}_{1}\right)+g\left(t+h_{1}, \bar{x}_{1}(t)\right)\right]+L_{1}\left(y(t)-C_{z} \bar{z}_{1}\left(t-\frac{h}{m}\right)\right), \\
& \dot{\bar{z}}_{j}(t)=A_{z} \bar{z}_{j}(t)+B_{z}\left[u\left(t-\bar{h}_{j}\right)+g\left(t+h_{j}, \bar{x}_{j}(t)\right)\right]+L_{j}\left(z_{j-1}(t)-\bar{z}_{j}\left(t-\frac{h}{m}\right)\right),
\end{aligned}
$$

for $j=2, \ldots, m$, being $L_{1} \in \mathbb{R}^{n_{z} \times q}, L_{j} \in \mathbb{R}^{n_{z} \times n_{z}}$ and $\bar{h}_{j}=h-h_{j}=\left(1-\frac{j}{m}\right) h$. Note that the particular case $m=1$ is feasible and then the observer is simply given by (8). Let us define the prediction error $\tilde{z}_{j}(t)=\left[\tilde{x}_{j}^{T}(t), \tilde{\xi}_{j}(t)\right]^{T}$, by

$$
\tilde{z}_{j}(t)=z(t)-\bar{z}_{j}\left(t-h_{j}\right)
$$

so that $\tilde{z}_{j}(t) \rightarrow 0$ implies $\bar{z}(t) \rightarrow z\left(t+h_{j}\right)$, as discussed above. Differentiating (10), using (6)-(9) and the Newton-Leibniz formula to rewrite $u(t-\tau(t))=u(t-h)-\mathcal{I}(\dot{u})$ 
with $\mathcal{I}(\phi) \triangleq \int_{t-\tau(t)}^{t-h} \phi(s) \mathrm{d} s$, the error dynamics satisfies ${ }^{1}$

$$
\begin{aligned}
& \dot{\tilde{z}}_{1}(t)=A_{z} \tilde{z}_{1}(t)-L_{1} C_{z} \tilde{z}_{1}\left(t-\frac{h}{m}\right)-B_{z} \mathcal{I}(\dot{u})+B_{z} \delta g_{1} \\
& \dot{\tilde{z}}_{j}(t)=A_{z} \tilde{z}_{j}(t)-L_{j} \tilde{z}_{j}\left(t-\frac{h}{m}\right)+L_{j} \tilde{z}_{j-1}\left(t-\frac{h}{m}\right)-B_{z} \mathcal{I}(\dot{u})+B_{z} \delta g_{j}
\end{aligned}
$$

where

$$
\delta g_{j}=g(t, x(t))-g\left(t, \bar{x}_{j}\left(t-h_{j}\right)\right) .
$$

Now, the proposed control law

$$
u(t)=-K \bar{x}_{m}(t)-g\left(t+h, \bar{x}_{m}(t)\right)-H \bar{\xi}_{m}(t),
$$

with $K \in \mathbb{R}^{1 \times n}$, is composed of three terms, the first two providing internal stability and the third one mitigating the effect of the disturbance. Note that (14) is a slight departure from the control law proposed in (Sanz, García, Fridman, \& Albertos, 2017), in which the nonlinear term was neglected.

Delaying (14) by $h$ units of time and using (10) with $j=m$, one can prove that the following holds

$$
u(t-h)=-K x(t)-w(t)-g\left(t, \bar{x}_{m}(t-h)\right)+F \tilde{z}_{m}(t) .
$$

where $F \triangleq[K, H]$. Using again the Newton-Leibniz equation into (1) and plugging (15) in, yields

$$
\dot{x}(t)=(A-B K) x(t)+B F \tilde{z}_{m}(t)-B \mathcal{I}(\dot{u})+B \delta g_{m} .
$$

\section{Closed-loop analysis}

Let us define $\mu(t)=\left[x^{T}(t), \tilde{z}_{1}^{T}(t), \ldots, \tilde{z}_{m}^{T}(t)\right]^{T} \in \mathbb{R}^{N}$ as an augmented state, where $N=n+m \cdot n_{z}$, and whose dynamics can be obtained from (11)-(12) and (16) as

$$
\dot{\mu}(t)=A_{0} \mu(t)+A_{1} \mu\left(t-\frac{h}{m}\right)-\Gamma_{0} \mathcal{I}(\dot{u})+\Gamma_{1} \delta g
$$

where $\delta g=\left[\delta g_{1}, \ldots, \delta g_{m}\right]^{T}$ and

$$
A_{0}=\left[\begin{array}{ccccc}
A-B K & 0 & \ldots & 0 & B F \\
0 & A_{z} & \ddots & \ddots & 0 \\
\vdots & \ddots & \ddots & \ddots & \vdots \\
0 & \ddots & \ddots & \ddots & 0 \\
0 & 0 & \ldots & 0 & A_{z}
\end{array}\right], \quad A_{1}=\left[\begin{array}{ccccc}
0 & 0 & \ldots & 0 & 0 \\
0 & -L_{1} C_{z} & \ddots & \ddots & 0 \\
\vdots & L_{2} & -L_{2} & \ddots & \vdots \\
0 & \ddots & \ddots & \ddots & 0 \\
0 & 0 & \ldots & L_{m} & -L_{m}
\end{array}\right],
$$

\footnotetext{
${ }^{1}$ The equality $z_{j-1}\left(t-h_{j}\right)-\bar{z}\left(t-h / m-h_{j}\right)=\tilde{z}_{j}(t-h / m)-\tilde{z}_{j-1}(t-h / m)$ was used to derive (12), which can be obtained by subtracting $\tilde{z}_{j}(t)$ and $\tilde{z}_{j-1}(t)$ as defined in (10), delaying the resulting expression by $h / m$ units of time, and using the fact that $h_{j-1}+h / m=h_{j}$.
} 


$$
\Gamma_{0}=\left[\begin{array}{c}
B \\
B_{z} \\
\vdots \\
\vdots \\
B_{z}
\end{array}\right], \quad \Gamma_{1}=\left[\begin{array}{ccccc}
0 & 0 & \ldots & 0 & B \\
0 & B_{z} & \ddots & \ddots & 0 \\
\vdots & \ddots & \ddots & \ddots & \vdots \\
0 & \ddots & \ddots & \ddots & 0 \\
0 & 0 & \ldots & 0 & B_{z}
\end{array}\right]
$$

In order to derive stability conditions for $(17)$, the term $\mathcal{I}(\dot{u})$ should be rewritten as a function of the augmented state $\mu$. Similarly, the bounds on the uncertain terms should be also expressed in that form. This is done in the following propositions.

Proposition 1. The integral $\mathcal{I}(\dot{u})$ can be expressed as

$$
\mathcal{I}(\dot{u})=\Phi_{0} \int_{t-\eta(t)}^{t} \dot{\mu}(s) \mathrm{d} s-\Delta g-\varphi(t)
$$

where $\Phi_{0}=[-K, 0, \ldots, 0, F]$ and

$$
\begin{aligned}
\Delta g & =g\left(t, \bar{x}_{m}(t-h)\right)-g\left(t-\eta(t), \bar{x}_{m}(t-\tau(t))\right), \\
\varphi(t) & =\int_{t-\eta(t)}^{t} \dot{w}(\theta) \mathrm{d} \theta .
\end{aligned}
$$

Proof. Introducing the change of variables $s=\theta-h$, the integral term can be written as $\mathcal{I}(\dot{u})=\int_{t-\tau(t)}^{t-h} \dot{u}(s) \mathrm{d} s=\int_{t-\eta(t)}^{t} \dot{u}(\theta-h) \mathrm{d} \theta$. Differentiating (15) and plugging it into the integral expression just derived yields the desired result. Note that the term $\Delta g=\int_{t-\eta(t)}^{t} \frac{\mathrm{d} g}{\mathrm{~d} \theta}(\theta, \bar{x}(\theta-h)) \mathrm{d} \theta$ has been expanded for convenience.

Proposition 2. The following inequalities hold

$$
\begin{aligned}
& \mathcal{S}_{1} \triangleq \mu^{T}(t) M_{1} \mu(t)-|\delta g|^{2} \geq 0, \\
& \mathcal{S}_{2} \triangleq\left[\begin{array}{ll}
\mu^{T}(t) & \int_{t-\eta(t)}^{t} \dot{\mu}^{T}(s) \mathrm{d} s
\end{array}\right]\left[\begin{array}{cc}
M_{3} & -M_{3} \\
(*) & M_{2}+M_{3}
\end{array}\right]\left[\begin{array}{c}
\mu(t) \\
\int_{t-\eta(t)}^{t} \dot{\mu}(s) \mathrm{d} s
\end{array}\right]-|\Delta g|^{2} \geq 0,
\end{aligned}
$$

where $M_{1}=\operatorname{diag}\left\{0_{n}, \bar{M}_{1}, \ldots, \bar{M}_{1}\right\}$ and

$$
\begin{gathered}
\Phi_{1}^{T}=\left[I_{n}, 0_{n \times 1}\right], \quad \Phi_{2}^{T}=\left[I_{n}, 0_{n \times n_{z}}, \ldots, 0_{n \times n_{z}},-\Phi_{1}^{T}\right] \\
\bar{M}_{1}=c_{1}^{2} \Phi_{1} l l^{T} \Phi_{1}^{T}, \quad M_{2}=2 c_{1}^{2} \Phi_{2} l l^{T} \Phi_{2}^{T}, \quad M_{3}=2 c_{2}^{2} \eta^{2} \Phi_{2} l l^{T} \Phi_{2}^{T} .
\end{gathered}
$$

Proof. See Appendix A.

Plugging (18) into (17), the closed-loop dynamics can be rewritten as

$$
\dot{\mu}(t)=A_{0} \mu(t)+A_{1} \mu(t-h)-A_{2} \int_{t-\eta(t)}^{t} \dot{\mu}(s) \mathrm{d} s+\Gamma_{0} \Delta g+\Gamma_{1} \delta g+\Gamma_{0} \varphi(t),
$$


where

$$
A_{2}=\left[\begin{array}{ccccc}
-B K & 0 & \ldots & 0 & B F \\
-B_{z} K & 0 & \ldots & 0 & B_{z} F \\
\vdots & \vdots & \ddots & \vdots & \vdots \\
-B_{z} K & 0 & \ldots & 0 & B_{z} F \\
-B_{z} K & 0 & \ldots & 0 & B_{z} F
\end{array}\right]
$$

One can see that (19) is driven by $\varphi(t)$, which arises as a consequence of the unknown time-varying delay. Asymptotic stability to zero will only be possible if $\bar{\eta}=0$ or $\lim _{t \rightarrow \infty} \dot{w}(t)=0$. Otherwise, we look at a conveniently defined $L_{2}$-gain performance. For a given $\gamma>0$, let us introduce the performance index

$$
J=\int_{0}^{\infty} y^{T}(t) y(t)-\gamma^{2} \bar{\eta} \psi(t) \mathrm{d} t
$$

where $\psi(t)=\int_{t-\eta(t)}^{t} \dot{w}(s)^{2} \mathrm{~d} s>0$ for all $0 \neq \dot{w} \in L_{2}[0, \infty)$. Next, an auxiliary result is given in Lemma 2, followed by a sufficient criterion for the closed-loop stability in Theorem 1. The design problem is then solved in Theorem 2.

Lemma 2. Let us denote $\mu_{t}(\theta)=\mu(t+\theta), \theta \in[-h-\bar{\eta}, 0]$ and $\left\|\mu_{t}\right\|_{W}=$ $\max _{[-h-\bar{\eta}, 0]}\left|\mu_{t}\right|+\left\|\mu_{t}\right\|_{L_{2}[-h-\bar{\eta}, 0]}$. If there is a Lyapunov-Krasovskii functional satisfying

$$
\beta_{1}|\mu(t)|^{2} \leq V\left(t, \mu_{t}, \dot{\mu}_{t}\right) \leq \beta_{2}\left\|\mu_{t}\right\|_{W}^{2},
$$

with $\beta_{1}, \beta_{2}>0$, such that, along the solutions of (19), the inequality

$$
\dot{V}\left(t, \mu_{t}, \dot{\mu}_{t}\right)+2 \alpha V\left(t, \mu_{t}, \dot{\mu}_{t}\right)+y^{T}(t) y(t)-\gamma^{2} \bar{\eta} \psi(t) \leq 0
$$

holds locally, then (19) is internally exponentially stable with decay rate $\alpha$ and achieves performance $J<0$ for all $\psi(t) \neq 0$ and zero initial conditions. Furthermore, if $\bar{\eta}=0$, then $\mu(t)$ converges to zero.

Proof. Setting $\psi(t)=0$ in (21) leads to $\dot{V}+2 \alpha V \leq 0$ and thus $|\mu(t)|^{2} \leq \beta_{1}^{-1} V(t) \leq$ $\beta_{1}^{-1} V(0) e^{-2 \alpha t} \leq \beta_{2} \beta_{1}^{-1} e^{-2 \alpha t}\left\|\mu_{0}\right\|_{W}^{2}$, where the second inequality follows by the comparison principle. This proves the internal $\alpha$-exponential stability. On the other hand, integration from 0 to $\infty$ leads to $J<0$, provided that $\mu_{0}=0$ implies $V\left(0, \mu_{0}, \dot{\mu}_{0}\right)=0$. Finally, setting $\bar{\eta}=0$ in (21) also leads to $\dot{V}+2 \alpha V \leq 0$ and thus by the same arguments as above, the convergence of $\mu(t)$ to zero follows. For additional details see (Fridman, 2014b) and the references therein.

Theorem 1. Given scalars $\gamma>0$ and $\bar{h}, \bar{\eta} \geq 0$, let there exist positive definite matrices 
$P, Q, R, S \in \mathbb{R}^{N}$, full matrices $P_{2}, P_{3} \in \mathbb{R}^{N}$ and positive scalars $\lambda_{1}, \lambda_{2}$ such that

$$
\left[\begin{array}{ccccccc}
(1,1) & (1,2) & P_{2}^{T} A_{1}+R e^{-2 \alpha \bar{h}} & -P_{2}^{T} A_{2}-\lambda_{2} M_{3} & P_{2}^{T} \Gamma_{0} & P_{2}^{T} \Gamma_{1} & P_{2}^{T} \Gamma_{0} \\
(*) & (2,2) & P_{3}^{T} A_{1} & -P_{3}^{T} A_{2} & P_{3}^{T} \Gamma_{0} & P_{3}^{T} \Gamma_{1} & P_{3}^{T} \Gamma_{0} \\
(*) & (*) & -(S+R) e^{-2 \alpha \bar{h}} & 0 & 0 & 0 & 0 \\
(*) & (*) & (*) & (3,3) & 0 & 0 & 0 \\
(*) & (*) & (*) & (*) & -\lambda_{2} & 0 & 0 \\
(*) & (*) & (*) & (*) & (*) & -\lambda_{1} I_{m} & 0 \\
(*) & (*) & (*) & (*) & (*) & (*) & -\gamma^{2}
\end{array}\right]<0
$$

where

$$
\begin{aligned}
& (1,1)=A_{0}^{T} P_{2}+P_{2}^{T} A_{0}+S-R+\bar{\eta}^{2} Q+\lambda_{1} M_{1}+\lambda_{2} M_{3}, \\
& (1,2)=P-P_{2}^{T}+A_{0}^{T} P_{3}, \\
& (2,2)=-P_{3}-P_{3}^{T}+\bar{h}^{2} R \\
& (3,3)=-U e^{-2 \alpha \bar{\eta}}+\lambda_{2}\left(M_{2}+M_{3}\right) .
\end{aligned}
$$

Then the closed-loop composed of the plant (1)-(2), the observer (8)-(9) and the control law (14) is internally exponentially stable and achieves $J<0$ for all $\psi(t) \neq 0$, with zero initial conditions and for any $0 \leq h \leq \bar{h}$. Furthermore, if $\bar{\eta}=0$, then $\mu(t)$ converges to zero with decay rate $\alpha$.

Proof. Let us consider a Lyapunov-Krasovskii functional of the form (see e.g. Section 3.7 of (Fridman, 2014a))

$$
V(t)=\mu(t)^{T} P \mu(t)+V_{h}(t)+V_{\eta}(t),
$$

where

$$
\begin{aligned}
V_{h}(t) & =\int_{t-h}^{t} e^{-2 \alpha(s-t)} \mu^{T}(s) S \mu(s) \mathrm{d} s+h \int_{-h}^{0} \int_{t+\theta}^{t} e^{2 \alpha(s-t)} \dot{\mu}(s)^{T} R \dot{\mu}(s) \mathrm{d} s \\
V_{\eta}(t) & =\bar{\eta} \int_{-\bar{\eta}}^{0} \int_{t+\theta}^{t} e^{2 \alpha(s-t)} \dot{\mu}(s)^{T} Q \dot{\mu}(s),
\end{aligned}
$$

Differentiating (23) and using the descriptor method (Fridman, 2001), one has that

$$
\begin{aligned}
\dot{V}(t)= & 2 \mu^{T}(t) P \dot{\mu}(t)+\mu^{T}(t) S \mu(t)-e^{-2 \alpha h} \mu^{T}(t-h) S \mu(t-h)+h^{2} \dot{\mu}^{T}(t) R \dot{\mu}(t) \\
& -e^{-2 \alpha h} h \int_{t-h}^{t} \dot{\mu}^{T}(s) R \dot{\mu}(t) \mathrm{d} s+\bar{\eta}^{2} \dot{\mu}^{T}(t) Q \dot{\mu}(t) \\
& -e^{-2 \alpha \bar{\eta}} \bar{\eta}(\underbrace{\int_{t-\bar{\eta}}^{t-\eta(t)} \dot{\mu}^{T}(s) Q \dot{\mu}(t) \mathrm{d} s}_{\text {neglected }}+\int_{t-\eta(t)}^{t} \dot{\mu}^{T}(s) Q \dot{\mu}(t) \mathrm{d} s) \\
& +2\left[\mu^{T}(t) P_{2}^{T}+\dot{\mu}^{T}(t) P_{3}^{T}\right][\operatorname{RHS} \text { of }(19)-\dot{\mu}(t)]
\end{aligned}
$$

where the last term in (26) can be added as it is identically zero. Now, by Jensen's 
inequality, it follows that

$$
\begin{aligned}
-\bar{\eta} \int_{t-\eta(t)}^{t} \dot{\mu}^{T}(s) Q \dot{\mu}(t) \mathrm{d} s & \leq \int_{t-\eta(t)}^{t} \dot{\mu}^{T}(s) \mathrm{d} s Q \int_{t-\eta(t)}^{t} \dot{\mu}(t) \mathrm{d} s \\
-h \int_{t-h}^{t} \dot{\mu}^{T}(s) R \mu(t) \mathrm{d} s & \leq \int_{t-h}^{t} \dot{\mu}^{T}(s) \mathrm{d} s R \int_{t-h}^{t} \dot{\mu}(t) \mathrm{d} s \\
& =-[\mu(t)-\mu(t-h)] R[\mu(t)-\mu(t-h)]
\end{aligned}
$$

Then, using (26)-(28) and Jensen's inequality to bound $-\bar{\eta} \psi(t) \leq-\varphi(t)^{2}$, one can write

$$
\dot{V}(t)-2 \alpha V(t)+y^{T}(t) y(t)-\gamma^{2} \bar{\eta} \psi(t) \leq q^{T}(t) \Xi q(t),
$$

where

$$
\begin{gathered}
\Xi=\left[\begin{array}{ccccccc}
\Xi_{11} & \Xi_{12} & P_{2}^{T} A_{1}+R e^{-2 \alpha h} & -P_{2}^{T} A_{2} & P_{2}^{T} \Gamma_{0} & P_{2}^{T} \Gamma_{1} & P_{2}^{T} \Gamma_{0} \\
(*) & \Xi_{22} & P_{3}^{T} A_{1} & -P_{3}^{T} A_{2} & P_{3}^{T} \Gamma_{0} & P_{3}^{T} \Gamma_{1} & P_{3}^{T} \Gamma_{0} \\
(*) & (*) & -(S+R) e^{-2 \alpha h} & 0 & 0 & 0 & 0 \\
(*) & (*) & (*) & -U e^{-2 \alpha \bar{\eta}} & 0 & 0 & 0 \\
(*) & (*) & (*) & (*) & 0 & 0 & 0 \\
(*) & (*) & (*) & (*) & (*) & 0 & 0 \\
(*) & (*) & (*) & (*) & (*) & (*) & -\gamma^{2}
\end{array}\right], \\
\Xi_{11}=A_{0}^{T} P_{2}+P_{2}^{T} A_{0}+S-R+\bar{\eta}^{2} Q, \\
\Xi_{12}=P-P_{2}^{T}+A_{0}^{T} P_{3}, \\
\Xi_{22}=-P_{3}-P_{3}^{T}+h^{2} R,
\end{gathered}
$$

and

$$
q(t)=\operatorname{col}\left\{\mu(t), \dot{\mu}(t), \mu(t-h), \int_{t-\eta(t)}^{t} \mu(s) \mathrm{d} s, \Delta g, \delta g, \varphi(t)\right\} .
$$

To deal with the uncertain terms $\delta g$ and $\Delta g$, the $\mathcal{S}$-procedure is invoked. Given the quadratic forms $\mathcal{S}_{1}, \mathcal{S}_{2} \geq 0$ in Proposition 2, it is verified that $q^{T}(t) \Xi q(t) \leq 0$ if there exist scalars $\lambda_{1}, \lambda_{2}>0$ such that

$$
q^{T}(t) \Xi q(t)+\lambda_{1} \mathcal{S}_{1}+\lambda_{2} \mathcal{S}_{2} \leq 0
$$

Rearranging (31) into a matrix form leads to (22). Since the LMI is convex in $h$, its feasibility for $\bar{h}$ implies its feasibility for any $0 \leq h \leq \bar{h}$. Finally, if (22) holds then so does (29). Furthermore, since $V\left(t, \mu_{t}, \dot{\mu}_{t}\right)$ in (23) clearly satisfies the lower and upper bounds in Lemma 2, then the theorem follows.

Theorem 2. Given scalars $\gamma, \epsilon>0$ and $\bar{h}, \bar{\eta} \geq 0$, let there exist positive definite matrices $W \in \mathbb{R}^{n}, P, Q, R, S \in \mathbb{R}^{N}$, full matrices $X \in \mathbb{R}^{1 \times n}, P_{20} \in \mathbb{R}^{n \times n}$, $P_{21}, \ldots, P_{2 m} \in \mathbb{R}^{n_{z} \times n_{z}}, Y_{21} \in \mathbb{R}^{n_{z} \times 1}, Y_{22}, \ldots, Y_{2 m} \in \mathbb{R}^{n_{z} \times n_{z}}$ and positive scalars 
$\lambda_{1}, \lambda_{2}$ such that

$$
\begin{gathered}
\text { W } A^{T}+A W-X^{T} B^{T}-B X+2 \alpha W<0 \\
{\left[\begin{array}{ccccccc}
(1,1) & (1,2) & \mathcal{Y}+R e^{-2 \alpha \bar{h}} & -P_{2}^{T} A_{2}-\lambda_{2} M_{3} & P_{2}^{T} \Gamma_{0} & P_{2}^{T} \Gamma_{1} & P_{2}^{T} \Gamma_{0} \\
(*) & (2,2) & \epsilon \mathcal{Y} & -\epsilon P_{2}^{T} A_{2} & \epsilon P_{2}^{T} \Gamma_{0} & \epsilon P_{2}^{T} \Gamma_{1} & \epsilon P_{2}^{T} \Gamma_{0} \\
(*) & (*) & -(S+R) e^{-2 \alpha \bar{h}} & 0 & 0 & 0 & 0 \\
(*) & (*) & (*) & (3,3) & 0 & 0 & 0 \\
(*) & (*) & (*) & (*) & -\lambda_{2} & 0 & 0 \\
(*) & (*) & (*) & (*) & (*) & -\lambda_{1} I_{m} & 0 \\
(*) & (*) & (*) & (*) & (*) & (*) & -\gamma^{2}
\end{array}\right]<0}
\end{gathered}
$$

where

$$
\begin{aligned}
& (1,1)=A_{0}^{T} P_{2}+P_{2}^{T} A_{0}+S-R+\bar{\eta}^{2} Q+\lambda_{1} M_{1}+\lambda_{2} M_{3}, \\
& (1,2)=P-P_{2}^{T}+\epsilon A_{0}^{T} P_{2}, \\
& (2,2)=-\epsilon P_{2}-\epsilon P_{2}^{T}+\bar{h}^{2} R, \\
& (3,3)=-U e^{-2 \alpha \bar{\eta}}+\lambda_{2}\left(M_{2}+M_{3}\right),
\end{aligned}
$$

$$
\mathcal{Y}=\left[\begin{array}{ccccc}
0 & 0 & \ldots & 0 & 0 \\
0 & -Y_{1} C_{z} & \ddots & \ddots & 0 \\
\vdots & Y_{2} & -Y_{2} & \ddots & \vdots \\
0 & \ddots & \ddots & \ddots & 0 \\
0 & 0 & \ldots & Y_{m} & -Y_{m}
\end{array}\right]
$$

and $P_{2}=\operatorname{diag}\left\{P_{20}, \ldots, P_{2 m}\right\}$. Then the closed-loop composed of the plant (1)-(2), the observer (8)-(9) with $L_{j}=\left(P_{2 j}^{T}\right)^{-1} Y_{j}$ and the control law (14) with $K=X W^{-1}$ is internally asymptotically stable and achieves $J<0$ for all $\psi(t) \neq 0$, with zero initial conditions any for any $0 \leq h \leq \bar{h}$. Furthermore, if $\bar{\eta}=0$, then the closed-loop converges exponentially to zero with decay rate $\alpha$.

Proof. Given the complexity of linearizing (22) to obtain both $K$ and $L$ simultaneously, the matrix $K$ is simply computed to guarantee that the plant (1) under the controller $u(t)=-K x(t)-g(t, x(t))$ is $\alpha$-exponentially stable, which is guaranteed by (32). Now, let us consider as in (Shustin \& Fridman, 2007; Suplin, Fridman, \& Shaked, 2007) the simplifications $P_{3}=\epsilon P_{2}$, with $\epsilon>0$ a scalar tuning parameter and $P_{2}=\operatorname{diag}\left\{P_{20}, \ldots, P_{2 m}\right\}$. Defining $Y_{j}=P_{2 j}^{T} L_{j}$, for $j=1,2, \ldots, m, \mathcal{Y}=P_{2}^{T} A_{1}$, and after some straightforward manipulations, the LMI (22) is transformed into (33), which completes the proof.

\section{Simulations}

The proposed strategy is illustrated in this section with three examples. The first one is academic example to validate the theoretical results stated in Theorem 2 . The others are focused on physical systems to illustrate the usefulness of this approach. First, a 

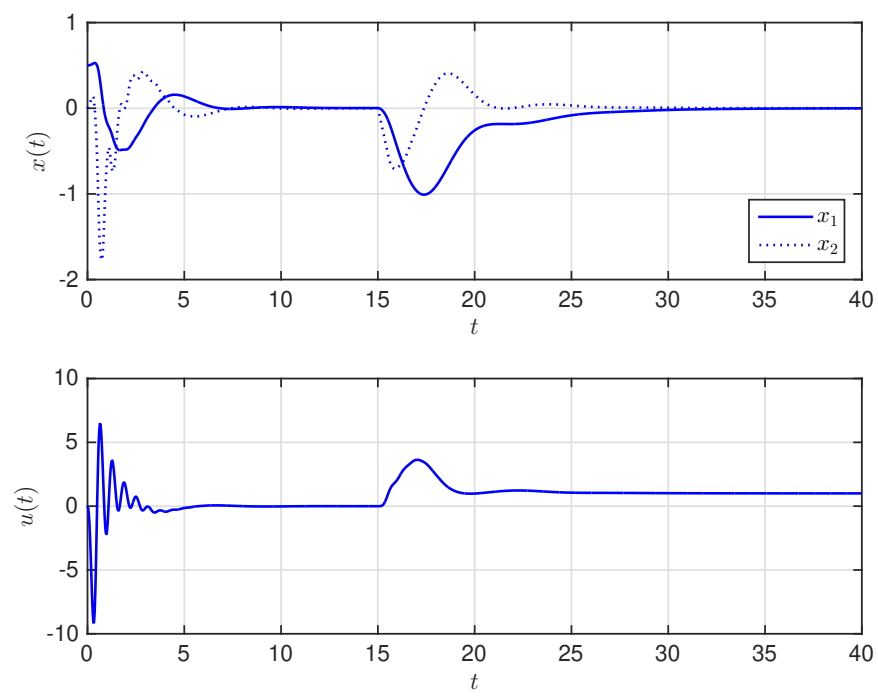

Figure 1. Example 1: state (top) starting from $x(0)=[0.5,0]^{T}$, with the observer starting from zero initial conditions and a disturbance $w(t)=-1, \forall t \geq 15$; control action (bottom)

servo motor with a nonlinear friction model is considered. Second, a simplified model of the longitudinal dynamics of an aircraft is also studied.

\subsection{Example 1}

Let us consider the following system

$$
\begin{aligned}
& \dot{x}_{1}(t)=x_{2}(t) \\
& \dot{x}_{2}(t)=x_{1}(t)+x_{1}^{2}(t) \sin t+u(t-\tau(t))+w(t)
\end{aligned}
$$

where $\tau(t)=0.2+0.05 \sin ^{2} t$ and $y(t)=x_{1}(t)$. The time delay function matches (3) with $h=0.2$ and $\bar{\eta}=0.05$. The system matrices

$$
A=\left[\begin{array}{ll}
0 & 1 \\
1 & 0
\end{array}\right], \quad B=\left[\begin{array}{l}
0 \\
1
\end{array}\right], \quad C=\left[\begin{array}{ll}
1 & 0
\end{array}\right],
$$

satisfy Assumption 1. It should be remarked that eig $(A)=\{-1,1\}$ and thus the openloop system is exponentially unstable. The nonlinearity $g(x, t)$ can be decomposed as the product of $g^{t}(t)=\sin t$ and $g^{x}(x)=x_{1}^{2}$. Clearly, $\left|g^{t}\left(t_{1}\right)-g^{t}\left(t_{2}\right)\right| \leq\left|t_{2}-t_{1}\right|, \forall t_{1}, t_{2} \geq$ 0. Also, $\left|g^{x}(y)-g^{x}(z)\right|=\left|\left(y_{1}+z_{1}\right)\left(y_{1}-z_{1}\right)\right| \leq \beta|[1,0,0](y-z)|, \forall\left|y_{1}+z_{1}\right| \leq \beta$. Therefore, Assumption 2 is satisfied with $c_{1}=1, c_{2}=1$ and $l^{T}=[\beta, 0,0]$ for all $\left|x_{1}\right| \leq \beta / 2$. The value of $\beta$ should be selected according to some design requirements and it is here arbitrarily chosen as $\beta=3$, which makes Assumption 2 to hold locally in the region $\mathcal{D}=\left\{x \in \mathbb{R}^{n}:\left|x_{1}\right| \leq 1.5\right\}$. The disturbance is considered to be constant, which satisfies Assumption 3 with $G=0$ and $H=1$. The observer (8)-(9) and the control law (14) are implemented with $m=2$. The gains $K$ and $L$ are designed using Theorem 2. The problem

$$
\min _{\gamma>0} \gamma \quad \text { subject to } \quad(32)-(33)
$$




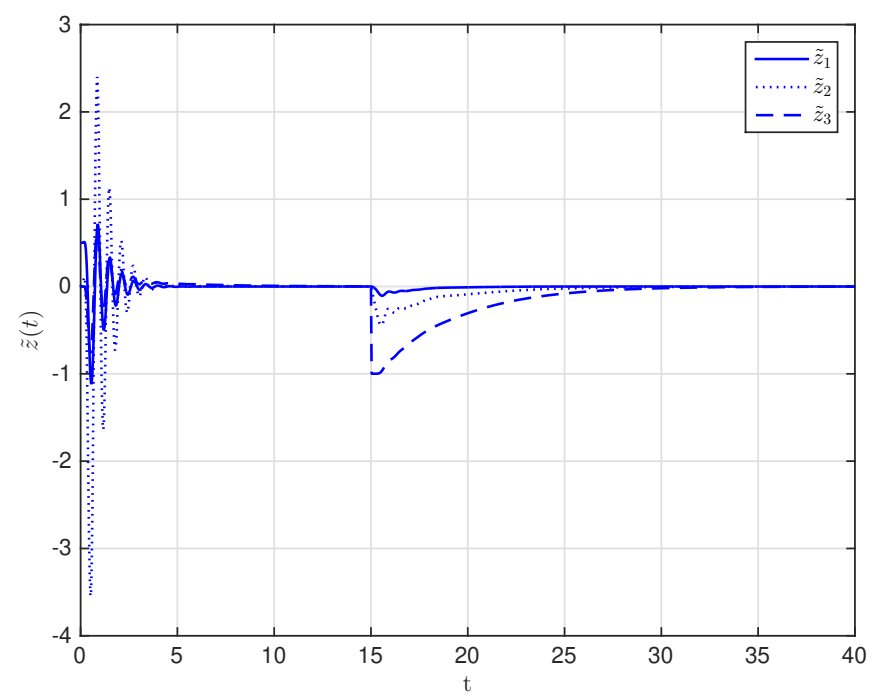

Figure 2. Example 1: observer error with the state starting from $x(0)=[0.5,0]^{T}$, the observer starting from zero initial conditions and a disturbance $w(t)=-1, \forall t \geq 15$

is solved using the Yalmip toolbox for Matlab. There are two parameters left to adjust, namely, the decay rate $\alpha \geq 0$ and auxiliary variable $\epsilon>0$. For a given value of $\alpha$, the problem (37) is solved for different values of $\epsilon$ and the one leading to the minimum $\gamma$ is taken as the optimal solution. This procedure is repeated for increasing values of $\alpha$ until the problem becomes unfeasible. In this example, we obtained $\gamma=63$, for $\alpha=0.15$ and $\epsilon=0.23$, leading to the gains

$$
K=\left[\begin{array}{ll}
2.54 & 1.18
\end{array}\right], \quad L_{1}=\left[\begin{array}{c}
8.92 \\
32.54 \\
10.50
\end{array}\right], \quad L_{2}=\left[\begin{array}{ccc}
6.65 & 0.01 & 0.51 \\
22.22 & 0.27 & 1.81 \\
4.15 & 0.21 & 0.41
\end{array}\right]
$$

A simulation with the system starting from $x(0)=[0.5,0]^{T}$ and a disturbance signal $w(t)=-1, \forall t \geq 15$ is carried out. The evolution of the system state and the control action are depicted in Fig. 1, where it can be seen that the state converges asymptotically to zero, as expected from Theorem 2 . The observer error is also shown in Fig. 2. It should be remarked that, in the example here considered, the previous work in (Sanz, García, Fridman, \& Albertos, 2017) fails to produce any stabilizing controller.

\subsection{Example 2}

Another example is considered, which consists of a servo positioning system governed by (Yao, Jiao, \& Ma, 2014)

$$
\theta_{1} \ddot{y}(t)=-F(\dot{y})-\theta_{4} \dot{y}(t)+\tau(t-h)+\tau_{L}(t),
$$

where $F(s)=\theta_{2} \tanh \left(c_{1} s\right)+\theta_{3}\left[\tanh \left(c_{2} s\right)-\tanh \left(c_{3} s\right)\right]$ is the friction model, $y$ is the motor rotation angle, $\tau$ is the motor torque, $\tau_{L}$ is a load disturbance torque and $\theta_{1}, \theta_{2}, \theta_{3}, \theta_{4}$ is a set of physical parameters. The following parameters $c_{1}=700, c_{2}=15$, $c_{3}=1.5, \theta_{1}=2.5 \cdot 10^{-3}, \theta_{2}=0.02, \theta_{3}=0.01$ and $\theta_{4}=0.205$ are given in (Yao et al., 2014). Only $x_{1}$ is assumed to be measured here and the model is modified to include 

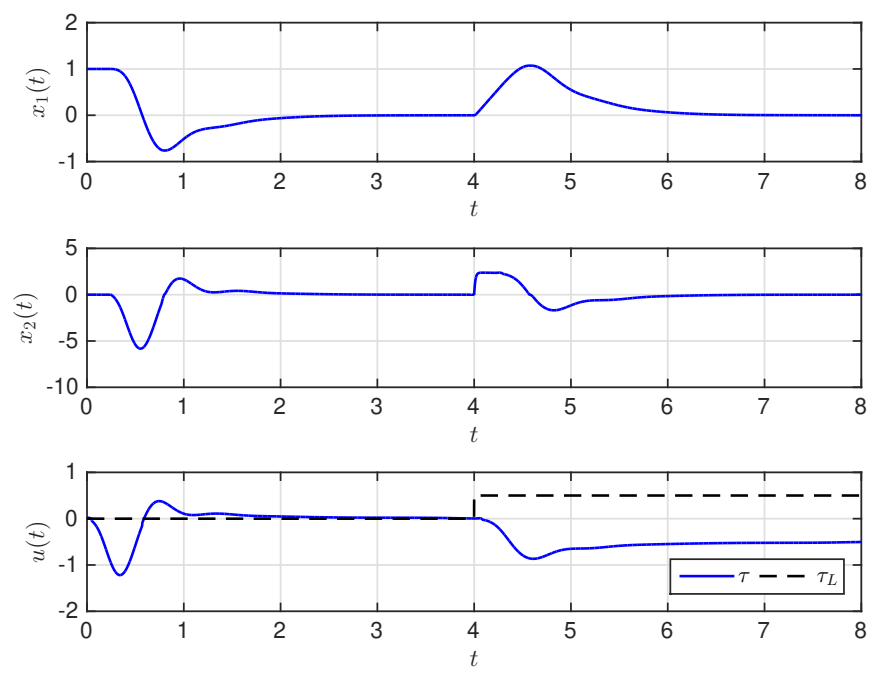

Figure 3. Example 2: State (top and center) starting from $x(0)=[1,0]^{T}$, with the observer starting from zero initial conditions and a disturbance $w(t)=0.5, \forall t \geq 4$; control action and disturbance (bottom)

an input delay of $h=0.1 \mathrm{~s}$. The system (38) is then written in the form of (1)-(2) with

$$
A=\left[\begin{array}{cc}
0 & 1 \\
0 & -\frac{\theta_{4}}{\theta_{1}}
\end{array}\right], \quad B=\left[\begin{array}{c}
0 \\
\frac{1}{\theta_{1}}
\end{array}\right], \quad C=\left[\begin{array}{ll}
1 & 0
\end{array}\right]
$$

and $g(t, x)=-F\left(x_{2}\right), w(t)=\tau_{L}(t)$. Assumption 1 is fulfilled by the triple $(A, B, C)$ and the nonlinearity satisfies Assumption 2 with $c_{1}=1, c_{2}=0$ and $l^{T}=\left[0, l_{2}\right]$, where $l_{2}$ is the Lipschitz constant that is computed numerically as $l_{2}=\sup _{s}\left|F^{\prime}(s)\right| \approx 14.13$. The disturbance is assumed to be a constant, which simulates a load attached to the motor shaft. Such disturbance satisfies Assumption 3 with $G=0$ and $H=1$.

Using Theorem 2 with $m=2, \alpha=2$ and $\epsilon=0.1$ yields the following gains

$$
L_{1}=\left[\begin{array}{c}
7.49 \\
-1.10 \\
2.71
\end{array}\right], \quad L_{2}=\left[\begin{array}{ccc}
6.47 & -0.33 & 1.55 \\
3.64 & -0.91 & 15.40 \\
0.97 & -0.11 & 2.51
\end{array}\right]
$$

and $K=\left[\begin{array}{ll}0.180 & -0.157\end{array}\right]$. A simulation with the system starting from $x=[1,0]^{T}$ is shown in Fig. 3. A constant load disturbance is introduced at $t=4 \mathrm{~s}$. One can see that system performance is fairly good in spite of the time delay and how the load disturbance is successfully identified and rejected.

\subsection{Example 3}

The aim of this example is to illustrate the rejection of a time-varying disturbance. The following model is an approximation of the longitudinal dynamics of $\mathrm{A} 4 \mathrm{D}$ aircraft 
at a flight condition of $15000 \mathrm{ft}$ and 0.9 Mach (Guo \& Chen, 2005)

$$
A=\left[\begin{array}{cccc}
-0.0605 & 32.3700 & 0 & 32.2000 \\
-0.0001 & -1.4750 & 1.0000 & 0 \\
-0.0111 & -34.7200 & -2.7930 & 0 \\
0 & 0 & 1.0000 & 0
\end{array}\right], \quad B=\left[\begin{array}{c}
0 \\
-0.1064 \\
-33.8000 \\
0
\end{array}\right]
$$

where the state $x=\left[x_{1}, x_{2}, x_{3}, x_{4}\right]$ is assumed to be measurable, $x_{1}$ is the forward velocity (ft/s), $x_{2}$ is the angle of attack ( $\mathrm{rad}$ ), $x_{3}$ is the pitching rate $\mathrm{rad} / \mathrm{s}, x_{4}$ is the pitch angle (rad) and $u$ is the elevator deflection (deg). As in (Guo \& Chen, 2005), the external disturbance is assumed to be a sinusoidal signal with frequency $5 \mathrm{rad} / \mathrm{s}$, described by Assumption 3 with

$$
H=\left[\begin{array}{ll}
25 & 0
\end{array}\right], \quad G=\left[\begin{array}{cc}
0 & 5 \\
-5 & 0
\end{array}\right] .
$$

The example is modified by including an input delay of $h=0.1 \mathrm{~s}$, which could be caused by a slow actuator dynamics. For the sake of comparison, the same $K$ as in (Guo \& Chen, 2005) is chosen, which is given by

$$
K=\left[\begin{array}{llll}
2.32 & 9.94 & 4.00 & 13.85
\end{array}\right]
$$

The observer gains were then chosen using Theorem 2, as explained in the previous example. Selecting $\alpha=0.5$ and $\epsilon=0.5$, the following gains were obtained

$$
L_{1}=\left[\begin{array}{cccc}
10.3 & 150.3 & 0.3 & -116.8 \\
0.2 & 17.3 & 0.9 & -12.2 \\
4.1 & 154.4 & 4.7 & -150.6 \\
0.4 & 17.5 & 0.9 & -11.7 \\
0.008 & -0.07 & -0.01 & 0.1 \\
0.003 & 0.06 & 0.02 & 0.1
\end{array}\right]
$$

and

$$
L_{2}=\left[\begin{array}{cccccc}
16.8 & 444.9 & -2.9 & -426.9 & -60.6 & 26.1 \\
0.5 & 35.2 & -0.5 & -22.5 & -23.5 & 5.7 \\
1.2 & 168.9 & -0.05 & -122.8 & -10.6 & 35.4 \\
0.8 & 41.5 & -0.5 & -28.6 & -24.5 & 6.1 \\
-0.005 & -0.5 & 0.004 & 0.3 & 0.4 & -0.06 \\
0.01 & 0.4 & -0.005 & -0.3 & -0.3 & 0.1
\end{array}\right]
$$

A simulation shows that the strategy reported in (Guo \& Chen, 2005) becomes unstable when the input delay $h=0.1 \mathrm{~s}$ is introduced. The results of the strategy proposed in this paper are shown in Fig. 4. One can see that, although the performance is obviously degraded, stability is preserved and the sinusoidal disturbance is rejected in spite of the delay. 

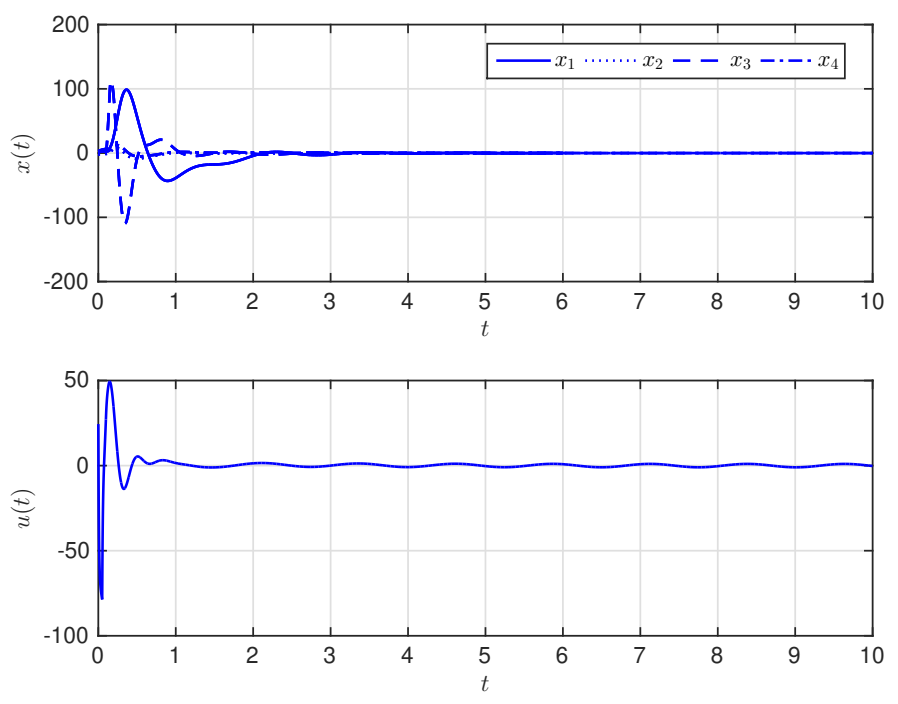

Figure 4. Example 3: State (top) starting from $x(0)=[2,-2,3,2]^{T}$, with the observer starting from zero initial conditions and a disturbance $w(t)=\sin 5 t, \forall t \geq 0$; control action (bottom)

\section{Conclusions}

A robust control strategy for a class of nonlinear systems with time-varying input delay was proposed. This strategy makes use of sequential predictors whose implementation is straightforward, in contrast to prediction-based controllers. Nonlinearities have been considered, which is an open problem in the context of sequential predictors. Furthermore, a design methodology by means of linear matrix inequalities has been derived. The design procedure has been illustrated with a numerical example.

Simulations show that the LMI design conditions are quite conservative. This is due to the restrictions imposed in the decision variables in order to derive computable design criteria. Therefore, other design procedures and/or Lyapunov-Krasovskii functionals that introduce less conservatism could be investigated in the future. Simulations also point out the so-called peaking phenomenon. This is a well-known behavior that can cause instability of nonlinear systems. Therefore, future research may also be focused on mitigating this effect by using saturation functions.

\section{Acknowledgements}

This work was partially supported by: Ministerio de Economía y Competitividad (TIN2017-86520-C3-1-R); Universitat Politècnica de València (16/17 UPV Mobility Award and FPI-UPV 2014 PhD Grant); and Israel Science Foundation (grant No. $1128 / 14)$.

\section{References}

Ahmed-Ali, T., Cherrier, E., \& Lamnabhi-Lagarrigue, F. (2012). Cascade high gain predictors for a class of nonlinear systems. IEEE Transactions on Automatic Control, 57(1), 221-226.

Artstein, Z. (1982). Linear systems with delayed controls: a reduction. Automatic Control, IEEE Transactions on, 27(4), 869-879. 
Basturk, H. I. (2017). Cancellation of unmatched biased sinusoidal disturbances for unknown LTI systems in the presence of state delay. Automatica, 76, 169-176.

Basturk, H. I., \& Krstic, M. (2015). Adaptive sinusoidal disturbance cancellation for unknown LTI systems despite input delay. Automatica, 58, 131-138.

Bekiaris-Liberis, N., \& Krstic, M. (2012). Compensation of time-varying input and state delays for nonlinear systems. Journal of Dynamic Systems, Measurement, and Control, 134(1), 011009.

Besançon, G., Georges, D., \& Benayache, Z. (2007). Asymptotic state prediction for continuous-time systems with delayed input and application to control. In 2007 European Control conference (ECC) (pp. 1786-1791).

Engelborghs, K., Dambrine, M., \& Roose, D. (2001). Limitations of a class of stabilization methods for delay systems. IEEE Transactions on Automatic Control, 46(2), 336-339.

Fridman, E. (2001). New lyapunov-krasovskii functionals for stability of linear retarded and neutral type systems. Systems \& Control Letters, 43(4), 309-319.

Fridman, E. (2014a). Introduction to time-delay systems: Analysis and control. Springer.

Fridman, E. (2014b). Tutorial on lyapunov-based methods for time-delay systems. European Journal of Control, 20(6), 271-283.

Furtat, I., Fridman, E., \& Fradkov, A. (2018). Disturbance compensation with finite spectrum assignment for plants with input delay. IEEE Transactions on Automatic Control.

Germani, A., Manes, C., \& Pepe, P. (2002). A new approach to state observation of nonlinear systems with delayed output. IEEE Transactions on Automatic Control, 47(1), 96-101.

Guo, L., \& Chen, W.-H. (2005). Disturbance attenuation and rejection for systems with nonlinearity via DOBC approach. International Journal of Robust and Nonlinear Control, $15(3), 109-125$.

Karafyllis, I., \& Krstic, M. (2017). Predictor feedback for delay systems: Implementations and approximations. Springer.

Krstic, M. (2008). Lyapunov tools for predictor feedbacks for delay systems: Inverse optimality and robustness to delay mismatch. Automatica, 44(11), 2930-2935.

Léchappé, V., Moulay, E., \& Plestan, F. (2016). Dynamic observation-prediction for LTI systems with a time-varying delay in the input. In Decision and Control (CDC), 2016 IEEE 55th conference on (pp. 2302-2307).

Léchappé, V., Moulay, E., Plestan, F., Glumineau, A., \& Chriette, A. (2015). New predictive scheme for the control of LTI systems with input delay and unknown disturbances. Automatica, 52, 179-184.

Manitius, A., \& Olbrot, A. W. (1979). Finite spectrum assignment problem for systems with delays. Automatic Control, IEEE Transactions on, 24(4), 541-552.

Mazenc, F., \& Malisoff, M. (2016). New prediction approach for stabilizing time-varying systems under time-varying input delay. In Decision and Control (CDC), 2016 IEEE 55th conference on (pp. 3178-3182).

Mondié, S., \& Michiels, W. (2003). Finite spectrum assignment of unstable time-delay systems with a safe implementation. IEEE Transactions on Automatic Control, 48(12), 2207-2212.

Najafi, M., Hosseinnia, S., Sheikholeslam, F., \& Karimadini, M. (2013). Closed-loop control of dead time systems via sequential sub-predictors. International Journal of Control, 86(4), 599-609.

Najafi, M., Sheikholeslam, F., Wang, Q.-G., \& Hosseinnia, S. (2014). Robust $H_{\infty}$ control of single input-delay systems based on sequential sub-predictors. IET Control Theory 86 Applications, 8(13), 1175-1184.

Sanz, R., Garcia, P., \& Albertos, P. (2016). Enhanced disturbance rejection for a predictorbased control of LTI systems with input delay. Automatica, 72, 205-208.

Sanz, R., García, P., \& Albertos, P. (2017). A generalized smith predictor for unstable timedelay siso systems. ISA transactions.

Sanz, R., García, P., Fridman, E., \& Albertos, P. (2017). A predictive extended state observer for a class of nonlinear systems with input delay subject to external disturbances. In Decision and Control (CDC), 2017 IEEE 56th Conference on (pp. 4345-4350). 
Sanz, R., Garcia, P., Fridman, E., \& Albertos, P. (2018). Rejection of mismatched disturbances for systems with input delay via a predictive extended state observer. International Journal of Robust and Nonlinear Control.

Shustin, E., \& Fridman, E. (2007). On delay-derivative-dependent stability of systems with fast-varying delays. Automatica, 43(9), 1649-1655.

Smith, O. (1957). Closer control of loops with dead time. Chemical Engineering Progress, 53, $217-219$.

Suplin, V., Fridman, E., \& Shaked, U. (2007). Sampled-data $H_{\infty}$ control and filtering: Nonuniform uncertain sampling. Automatica, 43(6), 1072-1083.

Yao, J., Jiao, Z., \& Ma, D. (2014). RISE-based precision motion control of DC motors with continuous friction compensation. IEEE Transactions on Industrial Electronics, 61(12), $7067-7075$.

Zhong, Q.-C. (2004). On distributed delay in linear control laws-part I: discrete-delay implementations. IEEE Transactions on Automatic Control, $49(11), 2074-2080$.

\section{Appendix A. Proof of Proposition 2}

Taking the norm of (13) and using Lemma 1 with $t_{1}=t_{2}=t, x_{1}=x(t)$ and $x_{2}=$ $\bar{x}_{j}\left(t-h_{j}\right)$ yields

$$
\left|\delta g_{j}\right| \leq c_{1}\left|m^{T}\left(x(t)-\bar{x}_{j}\left(t-h_{j}\right)\right)\right|=c_{1}\left|m^{T} \tilde{x}_{j}(t)\right|=c_{1}\left|m^{T} \Phi_{1}^{T} \tilde{z}_{j}(t)\right|
$$

Taking squares on both sides of (A1) yields $\left|\delta g_{j}\right|^{2} \leq \tilde{z}_{j}^{T}(t) \bar{M}_{1} \tilde{z}_{j}(t)$. Since $|\delta g|^{2}=$ $\sum_{j=1}^{m}\left|\delta g_{j}\right|^{2} \leq \sum_{j=1}^{m} \tilde{z}_{j}^{T}(t) M_{1} \tilde{z}_{j}(t)$ and recalling that $\mu(t)=\left[x^{T}(t), \tilde{z}_{1}^{T}(t), \ldots, \tilde{z}_{m}^{T}(t)\right]^{T}$, then $\mathcal{S}_{1} \geq 0$ follows.

Now, using Lemma 1 with $t_{1}=t, t_{2}=t-\eta(t), x_{1}=\bar{x}_{m}(t-h)$ and $x_{2}=\bar{x}_{m}(t-\tau(t))$ yields

$$
|\Delta g| \leq c_{1}\left|m^{T}\left[\bar{x}_{m}(t-h)-\bar{x}_{m}(t-\tau(t))\right]\right|+c_{2} \eta\left|m^{T} \bar{x}_{m}(t-\tau(t))\right|
$$

Let us rewrite

$$
\bar{x}_{m}(t-h)-\bar{x}_{m}(t-\tau(t))=\int_{t-\tau(t)}^{t-h} \dot{\bar{x}}_{m}(\theta) \mathrm{d} \theta=\int_{t-\eta(t)}^{t} \dot{\bar{x}}_{m}(s-h) \mathrm{d} s
$$

where the change of variable $\theta=s-h$ was performed. From (10), noting that $h=h_{m}$, we have that

$$
\bar{x}_{m}(t-h)=x(t)-\tilde{x}_{m}(t)=x(t)-\Phi_{1}^{T} \tilde{z}_{m}(t)=\Phi_{2}^{T} \mu(t) .
$$

Plugging (A4) into (A3), it follows that

$$
\bar{x}_{m}(t-h)-\bar{x}_{m}(t-\tau(t))=\int_{t-\eta(t)}^{t} \Phi_{2}^{T} \dot{\mu}(s) \mathrm{d} s .
$$

On the other hand, delaying (A4) by $\eta(t)$ units of time leads to

$$
\bar{x}_{m}(t-\tau(t))=\Phi_{2}^{T} \mu(t-\eta(t)) .
$$


Plugging (A5)-(A6) into (A2) yields

$$
|\Delta g| \leq c_{1}\left|m^{T} \Phi_{2}^{T} \int_{t-\eta(t)}^{t} \dot{\mu}(\theta) \mathrm{d} \theta\right|+c_{2} \eta\left|m^{T} \Phi_{2}^{T} \mu(t-\eta(t))\right|,
$$

Squaring both sides of (A7) and using Young's inequality to bound the cross term leads to

$$
|\Delta g|^{2} \leq\left(\int_{t-\eta(t)}^{t} \dot{\mu}^{T}(\theta) \mathrm{d} \theta\right) M_{2}\left(\int_{t-\eta(t)}^{t} \dot{\mu}(\theta) \mathrm{d} \theta\right)+\mu^{T}(t-\eta(t)) M_{3} \mu(t-\eta(t)) .
$$

Finally, replacing $\mu(t-\eta(t))$ by $\mu(t)-\int_{t-\eta(t)}^{t} \dot{\mu}(s) \mathrm{d} \theta$ in (A8) yields $\mathcal{S}_{2} \geq 0$, which completes the proof. 